2 Ansätze zur Operationalisierung und deren Erprobung im Feldtest zu PISA 2000

\title{
2.1 Einleitung
}

In Kapitel I dieses Bandes wurde erläutert, was bei PISA unter fächerübergreifender Problemlösekompetenz verstanden wird. Darüber hinaus wurden zwei unterschiedliche Problemtypen beschrieben, bei denen das Wissen der Problemlöser verschiedene Rollen spielt und die sehr unterschiedliche Messansätze erforderlich machen: Die Kompetenz, analytische Probleme zu lösen, betrifft die intelligente Anwendung von Wissen und lässt sich mit Papier-und-Beistift-Verfahren erfassen. Die Kompetenz, dynamische Probleme zu lösen, betrifft dagegen - neben der intelligenten Anwendung von Wissen - auch den intelligenten und selbstreguliert-strategischen Erwerb von Wissen und macht zur Erfassung computergestützte Verfahren erforderlich.

Das deutsche PISA-Konsortium hat sich im Rahmen der PISA-2000-Studie die Aufgabe gestellt, beide Arten von Problemiösekompetenz zu operationalisieren, das heißt entsprechende Testaufgaben zu entwickeln und Skalen zur Messung von Problemlösekompetenzen zu etablieren. Im Vorgriff auf spätere Erhebungen im internationalen Kontext, die dann mit PISA 2003 teilweise realisiert werden konnten, sollte das nationale Testprogramm in diesem Sinne ergänzt werden. Auch wenn man dabei auf Erkenntnisse und Instrumente der Problemlöseforschung zurückgreifen konnte (siehe Kap. I), stellte doch die Erhebung fächerübergreifender Problemlösekompetenzen innerhalb einer groß angelegten Schulleistungsstudie ein Novum dar. So galt es zunächst zu prüfen, ob sich in diesem Kontext praktikable und auch psychometrisch hinreichend geeignete Instrumente konstruieren lassen. Die Expertengruppe „Problemlösen“ des deutschen PISA-Konsortiums ${ }^{\mathrm{I}}$ entschied sich, insgesamt sechs unterschiedliche Aufgabentypen zu erproben, von denen dann zwei in der Hauptstudie PISA 2000 eingesetzt werden sollten (dazu siehe Kap. 3 und 4). Die Erprobung fand im Rahmen des PISA-Feldtests im Jahr I999 statt.

Schon mit der Erprobung wurde versucht, die psychologischen und erziehungswissenschaftlichen Fragestellungen des Gesamtprojekts in einer ersten, vorläufigen Weise zu beantworten: 
(I) Lässt sich fächerübergreifendes Problemlösen von Intelligenz und von fachlichen Kompetenzen empirisch abgrenzen? Dies ist die Frage nach der diskriminanten Validität von Messinstrumenten, die theoretisch unterschiedliche Konstrukte erfassen.

(2) Konvergieren analytische und dynamische Aspekte der Problemlösekompetenz, ohne jedoch als identisch angesehen werden zu müssen? Dies ist die Frage nach der konvergenten Validität von Messinstrumenten, die theoretisch ähnliche Konstrukte erfassen.

(3) Gehen interindividuelle Unterschiede der Problemlösekompetenz zwischen Schülerinnen und Schülern eher $\mathrm{zu}$ Lasten persönlicher (kognitiver und sozioökonomischer) oder eher zu Lasten schulischer Faktoren?

Die verwendeten Operationalisierungsansätze sowie die Antworten auf die grundlegenden psychologischen Fragen (Fragen I und 2) sollen in diesem Kapitel vorgestellt werden. Für ergänzende Informationen sowie Ergebnisse zur dritten Fragestellung sei auf die ausführlichere Darstellung dieser Vorstudie bei Klieme u.a. (200I) verwiesen.

\subsection{Operationalisierung von Problemlösekompetenz}

Fächerübergreifende Problemlösekompetenz sollte sinnvollerweise durch Aufgaben operationalisiert werden, die einen möglichst weiten Bereich unterschiedlicher Problemstellungen umfassen. Um dies zu gewährleisten, wurde in Anlehnung an Unterscheidungsmerkmale für Problemstellungen, die in der kognitiven Psychologie diskutiert werden (vgl. Funke, 2003; Jonassen, 2000), das in Tabelle 2.I dargestellte Klassifikationssystem verwendet.

- Hauptunterscheidungsmerkmal ist - entsprechend den eingangs formulierten Fragen - der durch konkrete Aufgabenstellungen zu operationalisierende Problemtyp: analytisch versus dynamisch (Wirth \& Klieme, 2003). Analytische Probleme erfordern die Anwendung von Wissen, und der in der Problemstellung beschriebene Ausgangszustand enthält alle für die Lösung erforderlichen Informationen entweder explizit oder implizit, sodass sie vom Problemlöser erschlossen werden können. Sie lassen sich in Papier-und-Bleistift-Form bearbeiten und betreffen die „Projektaufgaben“, das „Analoge Problemlösen“ und die „Fehlersuche“. Dynamische Probleme erfordern zunächst den Erwerb von Wissen, welches anschließend dann zur Anwendung kommen kann. Der in der Problemstellung präsentierte Ausgangszustand enthält die für die Problemlösung erforderlichen Informationen nur implizit; sie müssen vom Problemlöser erst durch eigene hypothesentestende Handlungen identifiziert, das heißt generiert oder entdeckt, und integriert werden. Probleme dieses Typs werden in Form von interaktiven Computersimulationen präsentiert und betreffen das "Virtuelle Labor", das „Ökologie-Planspiel“ und das „Raumfahrtspiel“.

- Ein zweites Unterscheidungsmerkmal ist die Art des Zielzustands, der als Lösung des jeweiligen Problems erreicht werden soll: wohldefiniert versus offen (Dörner, 1976; McCarthy, I956). Wohldefiniert ist ein Zielzustand, wenn eine präzise beschreibbare
Problemlösung auf einem präzise beschreibbaren Lösungsweg angestrebt werden soll. Dies gilt für die „Projektaufgaben“, das „Analoge Problemlösen“ und das „Raumfahrtspiel“. Offen ist ein Zielzustand, wenn die Lösung des Problems und/oder der Lösungsweg weniger präzise beschrieben werden kann und das Ziel teilweise oder ganz durch den Problemlöser selbst zu definieren ist. Dies gilt für die "Fehlersuche“, das „Virtuelle Labor“ und das „Ökologie-Planspiel“.

- Ein drittes Unterscheidungsmerkmal ist der Problemkontext: curriculumbezogen versus schulbezogen versus nicht schulbezogen. Curriculumbezogen ist ein Problemkontext, wenn die Problemstellung eine Thematik betrifft, die Gegenstand des schulischen Unterrichts ist, ohne dass jedoch - um den fächerübergreifenden Charakter der Problemlöseaufgaben zu gewährleisten - unmittelbar ersichtlich sein muss, welches fachliche Wissen zur Lösung nutzbar ist. Dies gilt für das „Virtuelle Labor“ und teilweise für das „Ökologie-Planspiel“, selbstverständlich aber auch für Lese-, Mathematik- und Naturwissenschaftsaufgaben, die jedoch nicht fächerübergreifend konzipiert sind. Schulbezogen ist ein Problemkontext, wenn die Problemstellung eine Thematik betrifft, die im alltäglichen Leben in der Schule und ihrem unmittelbaren Umfeld verankert ist, ohne notwendig direkt Gegenstand des Unterrichts zu sein. Dies gilt für die „Projektaufgaben“ und teilweise für das „Ökologie-Planspiel“. Nichtschul-bezogen ist schließlich eine Thematik, die ein alltägliches Problem außerhalb der Schule betrifft. Dies gilt für das „Analoge Problemlösen“, die „Fehlersuche“ und das „Raumfahrtspiel“. Um möglichst umfassend alle so klassifizierten Aspekte des Problemlösens abzudecken, wurden auf der Grundlage von Paradigmen der psychologischen Problemlöseforschung sechs verschiedene Verfahren zum Teil neu entwickelt, zum Teil an die Anforderungen eines Large-Scale Assessments adaptiert. Die in Tabelle 2.I kiassifizierten Aufgabentypen zum fächerübergreifenden Problemlösen sollen nun im Folgenden näher beschrieben werden (vgl. Funke, 2003; Klieme, 2004; Klieme u.a., 200r; Wirth \& Klieme, 2003). Wenn man von dem Merkmal „Art des Zielzustands“ absieht, dann decken sie die sechs Hauptbereiche der Tabelle 2.I ab - allerdings mit Ausnahme des Bereichs curriculumbezogener analytischer Probleme mit wohldefiniertem Zielzustand. In diesen Bereich

\begin{tabular}{|c|c|c|c|c|}
\hline \multicolumn{5}{|c|}{ 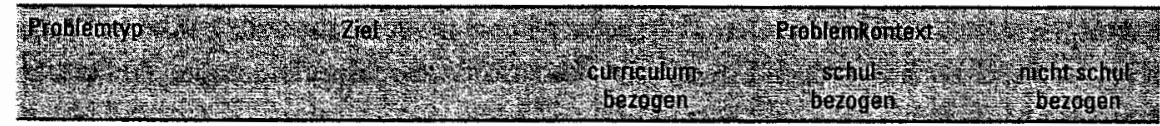 } \\
\hline \multirow[t]{2}{*}{$\begin{array}{l}\text { Dynamisches } \\
\text { Problem }\end{array}$} & Offen & \multicolumn{2}{|c|}{$\begin{array}{l}\text { Ökologie-Planspiel } \\
\text { Virtueiles Labor }\end{array}$} & \\
\hline & $\begin{array}{c}\text { Wohl- } \\
\text { definiert }\end{array}$ & & & Raumfahrtspiel \\
\hline \multirow{2}{*}{$\begin{array}{l}\text { Analytisches } \\
\text { Problem }\end{array}$} & Offen & & & Fehlersuche \\
\hline & $\begin{array}{l}\text { Wohl- } \\
\text { definiert }\end{array}$ & $\begin{array}{l}\text { Lesen, Mathematik, } \\
\text { Naturwissenschaften }\end{array}$ & $\begin{array}{l}\text { Projekt- } \\
\text { aufgaben }\end{array}$ & $\begin{array}{c}\text { Analoges } \\
\text { Problemlösen }\end{array}$ \\
\hline
\end{tabular}

Tabelle 2.1 Klassifikationssystem für Problemstellungen 
fallen die PISA-Literacy-Tests für Lesen, Mathematik und Naturwissenschaften, die in einem gewissen Sinne ebenfalls als Problemlöse-Test angesehen werden können.

\subsubsection{Aufgaben zum analytischen Problemlösen}

Projektaufgaben (Autoren: Klieme, Ebach u.a.)

Bei diesen Aufgaben handelt es sich um eine besondere Form „handlungsorientierter Diagnostik“: Ein „Projekt“ wie zum Beispiel die Organisation eines Klassenfestes wird in mehrere Arbeitsschritte aufgegliedert (Ziele klären - Informationen beschaffen - Planen - Entscheiden und Ausführen - Bewerten), zu denen jeweils einzelne Aufgaben gestellt werden. Der Test misst im Wesentlichen die Fähigkeit, Informationen zu erfassen, zu ordnen, aus unterschiedlichen Perspektiven zu bewerten und - unter Berücksichtigung multipler Zielsetzungen - Entscheidungen abzuleiten. Im PISA-Feldtest kommen zwei derartige "Projekte" aus thematisch unterschiedlichen Kontexten zum Einsatz. Gemäß Tabelle 2.I sind die Projektaufgaben als wohldefiniert und schulbezogen klassifiziert. Sie erfordern planendes und kombinatorisches Denken. (Die in der Haupterhebung PISA 2000 verwendete Variante des Aufgabentyps ist in Kap. 3 näher dargestellt.)

\section{Analoges Problemlösen (Autoren: Wirth, Klieme)}

Es werden mehrschrittige Planungs- und Ordnungsaufgaben gestellt, die jeweils in eine Alltagsgeschichte eingebunden sind. Zu jeder Aufgabe wird eine analoge Bezugsaufgabe mit ausgearbeiteter Lösung vorgegeben. Die Testperson muss die Beziehungen zwischen der ersten Aufgabe und der analogen Aufgabe erkennen und den Lösungsweg übertragen. Erfasst wird, wie gut der analoge Transfer beim Problemlösen gelingt. Gemäß Tabelle 2.I sind die Aufgaben zum analogen Problemlösen als wohldefiniert und nicht schulbezogen klassifiziert. Sie erfordern anialoges, schlussfolgerndes Denken.

Fehlersuche (Autoren: O'Neil, Baker u.a.; Adaptation: Klieme, Wirth)

Es wird die Skizze eines einfachen technischen Gerätes (z.B. einer Luftpumpe) vorgelegt. Der Test erfordert den Aufbau eines mentalen Modells seiner Funktionsweise, um auf dieser Basis dann Schlussfolgerungen über mögliche Funktionsfehler und deren Behebungen ziehen zu können. Gemäß Tabelle 2.I sind die Aufgaben zur Fehlersuche als offen und nicht schulbezogen klassifiziert. Sie erfordern schlussfolgerndes Denken in mentalen Modellen.

\subsubsection{Aufgaben zum dynamischen Problemlösen}

Virtuelles Labor (Autoren: Reimann, Schmitt)

In einem computersimulierten Labor soll die Testperson eine Serie von Experimenten durchführen und bestimmte Gesetzmäßigkeiten erschließen. Sie wählt dazu jeweils experimentelle Bedingungen aus und lässt sich das Experiment in einem Videoclip vorführen. Die Abfolge der Experimente und ihrer Resultate wird in einer Tabelle festge- halten. Auf der Basis dieser Informationen kann die Testperson dann Hypothesen formulieren und als richtig oder falsch bewerten. Die Experimentierumgebung besteht aus drei Komponenten: virtueller Experimentierraum (mit Videodarstellung), Ergebnistabelle und Hypothesenfenster. Erfasst wird, ob die im Gegenstandsbereich zentralen Hypothesen aufgestellt werden und wie systematisch die Testperson vorgeht. Gemäß Tabelle 2.I ist das Virtuelle Labor als offen und curriculumbezogen klassifiziert. Es erfordert Scientific Discovery as Dual Search (Klahr \& Dunbar, I988) in einem Hypothesen- und einem Experimentierraum.

Ökologie-Planspiel (Autor: Schrettenbrunner u.a.; Adaptation: Leutner)

Mit diesem Planspiel, das von Fachexperten als realitätsadäquat bewertet worden ist (vgl. Leutner \& Schrettenbrunner, I989), wird die Situation eines Agrarbetriebs simuliert, wobei Themen mehrerer schulischer Fächer angesprochen werden. Erfasst wird, wie effizient die Testperson das System exploriert, welches Wissen sie dabei erwirbt und wie gut sie in einem abschließenden Testdurchgang den Agrarbetrieb bewirtschaften kann. Gemäß Tabelle 2.I ist das Ökologie-Planspiel als offen klassifiziert. Es kann sowohl als curriculum- als auch als schulbezogen gelten, da in der Gestaltung des schulischen Alltags agrarökologische Fragen ebenfalls vorkommen können (z.B. als „Schulgarten“). Das System ist vergleichsweise komplex (viele quantitative Parameter, vernetzt, intransparent und einer Eigendynamik unterworfen) und repräsentiert damit die Forschungstradition des "komplexen Problemlösens" im Sinne von Dörner (1989). Gemäß dieser Tradition erfordert es Learning by Doing auf der Basis des Suchens und Sich-Bewegens in einem unendlichen Problemraum, der als naturalistisches Szenario eingekleidet ist.

Raumfahrtspiel (Autoren: Funke, Töpfer, Wagener)

In der Forschung zum "komplexen Problemlösen“ wurden nicht nur dynamische Systeme mit quantitativen Variablen untersucht, wie beispielsweise die zuvor beschriebene ökologische Simulation, sondern auch diskrete Systeme. Derartige Systeme können eine bestimmte Menge an Zuständen annehmen, die durch bestimmte Eingriffe (darstellbar als Betätigen von „Schaltern“) verändert und ineinander überführt werden können. Alltagsbeispiele sind Fahrkartenautomaten, Mobiltelefone oder andere elektronische $\mathrm{Ge}$ räte. Es können aber auch technische, ökonomische oder soziale Prozesse mit derartigen Modellen (so genannten "finiten Automaten") dargestellt werden (Buchner \& Funke, I993; Frensch \& Funke, I995). Für PISA wurde eine Variante entwickelt, die in ein Raumfahrtszenario eingebettet ist. Erfasst wird auch hier, wie effizient die Testperson das System exploriert, welches Wissen sie dabei erwirbt und mit welchem Erfolg sie abschließende Steuerungsaufgaben bewältigt. Gemäß Tabelle 2.I ist das Raumfahrtspiel als wohldefiniert und nicht schulbezogen klassifiziert. Auch dieses System erfordert das Suchen und Sich-Bewegen in einem Problemraum und Learning by Doing. Im Gegensatz zum Ökologie-Planspiel ist dieser Problemraum allerdings nicht unendlich, sondern endlich. (Die in der Haupterhebung PISA 2000 verwendete Variante des Raumfahrtspiels wird in Kap. 4 im Detail beschrieben.) 


\subsection{Empirische Erprobung im Feldtest}

Die im Abschnitt zuvor beschriebenen Messinstrumente wurden im 1999 durchgeführten PISA-Feldtest erprobt. Bei den Instrumenten zum fächerübergreifenden Problemlösen handelte es sich beim deutschen PISA-Feldtest um eine nationale Ergänzung des international eingesetzten Testmaterials, wozu für die Aufgaben zum analytischen Problemlösen der zweite Testtag genutzt wurde und für die Aufgaben zum dynamischen Problemlösen bei einer Teilstichprobe ein dritter Testtag erforderlich wurde.

\subsubsection{Zielsetzung und methodische Vorgehensweise}

Ziel des Feldtests war zunächst zu prüfen, ob die entwickelten bzw. adaptierten Instrumente praktikabel einsetzbar sind und für Assessmentzwecke hinreichend gute psychometrische Eigenschaften aufweisen. Zentrales weiteres Ziel war die Konstruktvalidierung der Instrumente. Darüber hinaus sollten erste Schritte unternommen werden, die Sensitivität von Maßen der Problemlösekompetenz für Effekte institutionalisierter Bildung zu untersuchen.

\section{Stichprobe, Design und Vorgehensweise}

Am PISA-Feldtest nahmen insgesamt nahezu 4.000 Schülerinnen und Schüler teil. In der Problemlöse-Teilstichprobe waren insgesamt $6_{54}$ Schülerinnen und Schüler aus I8 Schulen in vier Bundesländern einbezogen. Pro Schule waren Teilnehmer ausgewählt, die überwiegend der internationalen PISA-Zielgruppe der 15-Jährigen, zum geringeren Teil der deutschen Ergänzungspopulation von Schülern der 9. Jahrgangsstufe angehörten. Alle Schülerinnen und Schüler dieser Problemlöse-Stichprobe bearbeiteten - wie die übrigen Teilnehmer des PISA-Feldtests - an einem ersten Testtag die internationalen Aufgaben zum Lesen, zur Mathematik und zu den Naturwissenschaften sowie einen Begleitfragebogen, in dem unter anderem auch demographische Variablen wie zum Beispiel Geschlecht und sozioökonomischer Status erhoben wurden.

An einem zweiten Testtag wurden bei allen Teilnehmern nationale Ergänzungsaufgaben und Fragebögen eingesetzt. An diesem Tag bearbeitete die eine Hälfte der Problemlöse-Teilstichprobe die Projektaufgaben, die andere Hälfte die Aufgaben zum analogen Problemlösen. Hinzu kamen für alle Teilnehmer die Subtests „Figurale Analogien“ und „Wortanalogien“ des Kognitiven Fähigkeitstests (Heller, Gaedike, \& Weinläder, I9 85 ) als Maße der allgemeinen Intelligenz (Reasoning). Zudem wurde den Teilnehmern ein nationaler Ergänzungsfragebogen vorgelegt.

Ausschließlich für die I 8 Schulen der Problemlöse-Stichprobe wurde schließlich ein dritter Testtag organisiert. Mitarbeiter des Max-Planck-Instituts für Bildungsforschung bereisten, ausgerüstet mit einem Satz von 30 identisch konfigurierten Laptop-PCs, die Schulen und führten jeweils in einer Doppelstunde die Erhebungen durch. Diese bestanden im Wesentlichen aus den - um eine "Maus-Tracking“-Aufgabe zur Erfassung der Informationsverarbeitungsgeschwindigkeit ergänzten - drei computergestützten
Verfahren sowie aus der Aufgabe zur Fehlersuche, die einer ausführlichen mündlichen Instruktion bedurfte und daher nicht bereits am zweiten Testtag bearbeitet werden konnte. Ergänzend wurden per Fragebogen das thematische Interesse und das Vorwissen zu den Problemstellungen sowie die Computererfahrung erhoben. Nach einem ausbalancierten Design bearbeitete die Mehrheit der Teilnehmer je zwei dieser Tests in wechselnden Kombinationen, sodass jedes einzelne Verfahren von etwa I80 Schülern und jede Kombination von etwa 60 Schülem bearbeitet wurde ${ }^{2}$. Kleinere Teile der Problemlöse-Stichprobe bearbeiteten auch am dritten Testtag eines der schriftlichen Verfahren (Projektaufgabe bzw. analoges Problemlösen), sodass in Verbindung mit den Daten des zweiten Testtages auch Zusammenhänge zwischen diesen beiden Aufgabentypen untersucht werden konnten.

\section{Operationalisierung von Komponenten der Problemlösekompetenz}

Bei den schriftlichen Verfahren zum analytischen Problemlösen lässt sich Problemlösekompetenz operationalisieren als Anzahl der richtigen Antworten zu den einzelnen Aufgabenstellungen. Insofern gibt es jeweils ein Maß für die Projektaufgaben, für die Aufgaben zum analogen Problemlösen und für die Aufgaben zur Fehlersuche. Bei den computerbasierten Verfahren zum dynamischen Problemlösen lassen sich - entsprechend der zu Grunde liegenden Unterscheidung von Wissenserwerb und Wissensanwendung - dagegen mehrere Maße erzeugen. Die Testung erfolgte in drei Phasen: Exploration, Wissenstest, Steuerungstest. In der ersten Phase war das System zu explorieren, um zu verstehen, wie es funktioniert. Aus den Logfiles der Computerprogramme ließen sich Maße für die Effizienz des Explorationsverhaltens ableiten. In der zweiten Phase wurde schriftlich das in der Explorationsphase erworbene Wissen erfragt, sodass auch hier die Anzahl der richtigen Antworten das Maß der Wahl ist. In der Steuerungsphase schließlich, die nur beim Ökologie-Planspiel und beim Raumfahrtspiel sinnvoll erhoben werden konnte, ging es um die Anwendung des in der Explorationsphase erworbenen Wissens: Das jeweils simulierte System war durch geschickte Eingriffe auf einen bestimmten Zielzustand hin zu steuern, und aus den Logfiles der Computerprogramme ließ sich jeweils ein Maß für die Steuerungsleistung ableiten. Die Problemlösekompetenz wird beim Ökologie-Planspiel und beim Raumfahrtspiel demzufolge durch die drei Komponenten „Exploration“, „Wissen“ und „Steuern" repräsentiert, beim Virtuellen Labor nur durch die zwei Komponenten „Exploration“ und „Wissen“.

\subsubsection{Ergebnisse zur Einsetzbarkeit und zur Reliabilität der Instrumente}

Die den Einsatz der schriftlichen Instrumente zum analytischen Problemlösen betref fende Praktikabilität erwies sich erwartungsgemäß als unproblematisch. Der Einsatz der computerbasierten Instrumente zum dynamischen Problemlösen war dagegen mit einem durchaus als erheblich zu bezeichnenden, aber dennoch zu bewältigenden Aufwand verbunden, nicht zuletzt weil zur damaligen Zeit noch nicht alle Schulen über hinreichend geeignete Hardware verfügten und aus diesem Grund von den Testleitern mit- 


\begin{tabular}{|c|c|c|}
\hline Thstrument & twien: & abiti \\
\hline Projektaufgaben & 0,68 & 0,74 \\
\hline Analoges Problemlösen & 0,33 & 0,79 \\
\hline Fehlersuche & 0,36 & 0,63 \\
\hline $\begin{array}{l}\text { Wissenstest des } \\
\text { Raumfahrtspiels } \\
\text { Virtuellen Labors } \\
\text { Okologie-Planspiels }\end{array}$ & $\begin{array}{l}0,53 \\
0,50 \\
0,62\end{array}$ & $\begin{array}{l}0,82 \\
0,78 \\
0,63\end{array}$ \\
\hline
\end{tabular}

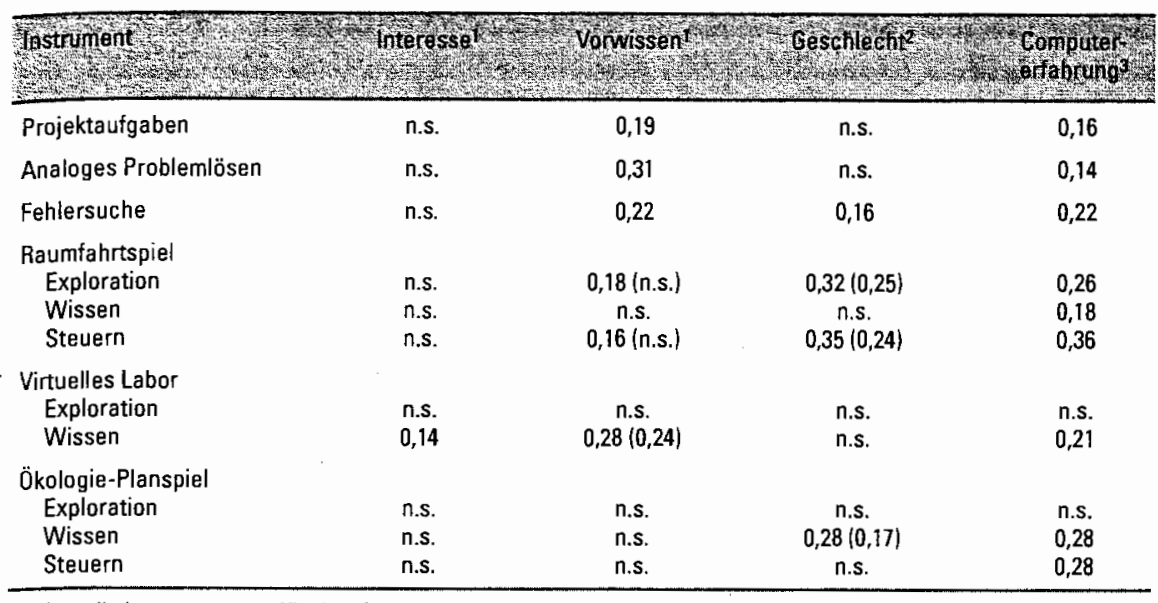

gebrachte Laptop-PCs auf- und abgebaut werden mussten. Außerdem gab es vereinzelt Systemausfälle, die dazu führten, dass die Logfiles der betroffenen Teilnehmer nicht gesichert werden konnten. Im Großen und Ganzen erwies sich dieser Aufwand jedoch noch als praktikabel, und der Datenverlust hielt sich in sehr engen Grenzen.

Tabelle 2.2 enthält grundlegende Informationen zu psychometrischen Eigenschaften der Problemlösemaße. Es zeigt sich, dass die Schwierigkeit (operationalisiert als mittlere Lösungswahrscheinlichkeit von Items) der Skalen zum analytischen Problemlösen und der Wissensskalen zum dynamischen Problemlösen in einem akzeptablen mittleren Bereich liegen, wobei das analoge Problemlösen und die Fehlersuche als etwas schwieriger und die Projektaufgaben und das Geographie-Planspiel als etwas leichter auffallen. Darüber hinaus ist festzustellen, dass die Reliabilität der Skalen (operationalisiert als Cronbachs $\alpha$ ) ebenfalls akzeptable Werte erreicht, wobei die Fehlersuche und das Geographie-Planspiel im Vergleich zu den anderen Skalen eine etwas geringere Zuverlässigkeit aufweisen.

Tabelle 2.3 enthält Informationen zur Frage, inwieweit die Skalenwerte der Problem. löse-Skalen im Sinne eines "Bias" durch andere konstruktferne Variablen verzerrt sind. So könnte man zum Beispiel erwarten, dass Testteilnehmer mit hoher Computererfahrung bei den computergestützten Verfahren im Vorteil sein könnten. Ein erster Blick in die letzte Spalte von Tabelle 2.3 zeigt tatsächlich für die computergestützten Verfahren eine etwas höhere Korrelation als für die schriftlichen Verfahren, wobei die Explorationskomponenten des Virtuellen Labors und des Geographie-Planspiels nicht statistisch signifikant mit der Computererfahrung korrelieren. Allerdings ist der Unterschied bei den signifikanten Korrelationen nur marginal und erreicht erst bei der Steuerkomponente des Raumfahrtspiels eine Größenordnung, die zwar im Vergleich zu den Projektaufgaben und dem analogen Problemlösen statistisch signifikant ist, nicht aber im Vergleich zur Fehlersuche. Im Übrigen ist die Computererfahrung im Sinne einer positiven Korrelation mit Intelligenz konfundiert. Kontrolliert man die Intelligenz, dann sinken die (Partial-)Korrelationen für die schriftlichen Verfahren auf unter .I5 und bei den computerbasierten Verfahren immerhin auf Werte zwischen .I5 und .30. Akzeptabel sind auch die geschlechterspezifischen Unterschiede der Problemlösekompetenz. Zwar

Jeweils instrumentspezifisch erfragt

3 Per Fragebogen erfragt.

n.s.: nicht statistisch signifikante Korrelationen. Bei den computerbasierten Verfahren sind in Klammern die Partialkorrelationen bei Kontroile der Computererfahrung angegeben.

Tabelle 2.3 Korrelation der Problemlöseskalen mit konstruktfremden Variablen

schneiden Jungen bei der Explorations- und der Steuerungskomponente des Raumfahrtspiels und bei der Wissenskomponente des Ökologie-Planspiels etwas besser ab als Mädchen, der Unterschied verschwindet aber weitgehend, wenn auch nicht vollständig, wenn die Computererfahrung kontrolliert wird.

Bemerkenswert und erfreulich ist (vgl. Tab. 2.3), dass die von den Testteilnehmern bei den computerbasierten Instrumenten erreichten Skalenwerte mit Ausnahme der Wissenskomponente beim Virtuellen Labor mit dem verfahrensspezifisch erfragten thematischen Interesse nicht korrelieren und dass sich auch beim verfahrensspezifisch erfragten Vorwissen, ebenfalls mit der Ausnahme der Wissenskomponente des Virtuellen Labors, keine nennenswerten Korrelationen zeigen: Die niedrigen, aber statistisch signifikanten Korrelationen des Vorwissens mit der Explorations- und der Steuerungskomponente des Raumfahrtspiels verschwinden, wenn Computererfahrung kontrolliert wird. Für die schriftlichen Verfahren zeigen sich ebenfalls keine Korrelationen mit dem thematischen Interesse, wohl aber beim Vorwissen und dort insbesondere beim analogen Problemlösen.

Als Fazit ist festzustellen, dass die Adaptation von Verfahren der Problemlöseforschung für das Assessment von Problemlösekompetenz in einer großen Stichprobe durchaus als gelungen bezeichnet werden kann. Die Verfahren haben einen angemessenen Schwierigkeitsgrad und eine weitgehend gute Reliabilität. Zusammenhänge mit Interesse, Vorwissen, Geschlecht und Computererfahrung sind mit wenigen Ausnah- 
men nicht als bedeutsam anzusehen und dürften daher die Fairness der Testinstrumente nicht gefährden.

\subsubsection{Ergehnisse zur konvergenten und diskriminanten Konstruktvalidität}

\section{der Instrumente}

Im Folgenden werden zunächst die computerbasierten Verfahren anhand von Pfad modellen daraufhin untersucht, ob sie tatsächlich über die schriftlichen Verfahren hinausgehen und nicht nur die intelligente Anwendung, sondern auch den intelligenten Erwerb von Wissen erfassen. Im nächsten Schritt wird anhand von Strukturmodellen geprüft, ob dynamisches und analytisches Problemlösen unterschiedliche Dimensionen der Problemlösekompetenz darstellen, die sich von Intelligenz abgrenzen lassen Schließlich wird im dritten Schritt ebenfalls anhand von Strukturmodellen geprüft, inwieweit sich die Dimensionen der Problemlösekompetenz auch dann noch voneinander abgrenzen lassen, wenn die fachlichen Kompetenzen bezüglich Lesen, Mathematik und Naturwissenschaften mit in das Modell aufgenommen werden. Abschließend werden die fachlichen und die Problemlösekompetenzen anhand einer multidimensionalen Skalierung auf ihre Struktur hin untersucht.

$\mathrm{Zu}$ beachten ist, dass das Virtuelle Labor bei den folgenden Analysen nicht weiter berücksichtigt wird: Zum einen zeigt es als einziges der computergestützten Verfahren wenig wünschenswerte Korrelation mit thematischem Interesse und Vorwissen. Zum an deren besteht beim Virtuellen Labor im Gegensatz zu den anderen beiden Verfahren keine Möglichkeit, die Steuerungskomponente der dynamischen Problemlösekompetenz zu erfassen, sodass es in Strukturmodellen schon allein durch die Art der Indikatorvariablen aus dem Rahmen fallen würde.

\section{Pfadmodelle der computerbasierten Verfahren}

Fächerübergreifendes Problemlösen wurde eingangs als intelligentes Anwenden von Wissen konzeptualisiert, wobei bei dynamischen Problemen im Gegensatz zu analytischen Problemen noch der intelligente Erwerb von Wissen innerhalb der Problemsituation konstituierend hinzutritt (Learning by Doing). Intelligenz sollte also bei allen Problemlöseleistungen eine gewisse Rolle spielen, wobei diese Rolle bei analytischen Problemen größer sein dürfte als bei dynamischen Problemen, allein schon aufgrund desselben Präsentationsformats als Papier-und-Bleistift-Test. Die Rohkorrelationen zeigen genau dieses Muster: Die Korrelation der Intelligenz mit den schriftlichen Testverfahren beträgt . $70<\mathrm{r}<.72$, während die Korrelation der Intelligenz mit den Komponenten der Problemlösekompetenzen beim Raumfahrtspiel im Bereich. $45<\mathrm{r}<.58$ und beim Ökologie-Planspiel im Bereich $.23<\mathrm{r}<.58$ liegt. Bemerkenswert ist, dass bei den computerbasierten Verfahren jeweils die höchste Korrelation (in beiden Fällen $r=.58$ ) bei der Wissenskomponente auftritt, die ebenfalls schriftlich (als Multiple-Choice-Tes am Computer) erfasst wurde und im Hinblick auf die Reliabilität der Skalenwerte mit den schriftlichen Verfahren vergleichbar ist (vgl. Tab. 2.2). Intelligenz spielt also sowohl beim analytischen als auch beim dynamischen Problemlösen eine maßgebliche Rolle, die allerdings beim analytischen Problemlösen offensichtlicher ist als beim dynamischen Problemlösen. Dies ist ganz im Einklang mit der Elshout-Raaheim-Hypothese (Leutner, 2002; vgl. Kap. I, in diesem Band), nach der Intelligenz beim Problemlösen vor allem dann deutlich ins Spiel kommt, wenn zur Lösung des Problems auf vorhandenes Wissen zurückgegriffen werden kann („Problemlösen als intelligente Anwendung von Wissen "). Anders sieht dies dagegen beim dynamischen Problemlösen aus: Hier scheinen die Testpersonen (wie bei der Konstruktion der Verfahren bezweckt) tatsächlich weniger auf vorhandenes Wissen zurückgreifen zu können (das müssen sie sich im Rahmen der Exploration erst erarbeiten), was sich ganz im Sinne der Elshout-RaaheimHypothese in einer insgesamt reduzierten Korrelation mit Intelligenz niederzuschlagen scheint.

Die Pfadmodelle in den Abbildungen 2.I und 2.2 zeigen für beide dynamische Verfahren, dass Intelligenz einen starken direkten Pfad auf das am Ende der Systemexploration abrufbare Wissen hat, dass bei beiden Verfahren aber auch ein indirekter Pfad über die Exploration geht: Wer intelligent ist, exploriert effizient und erwirbt auf diese Weise

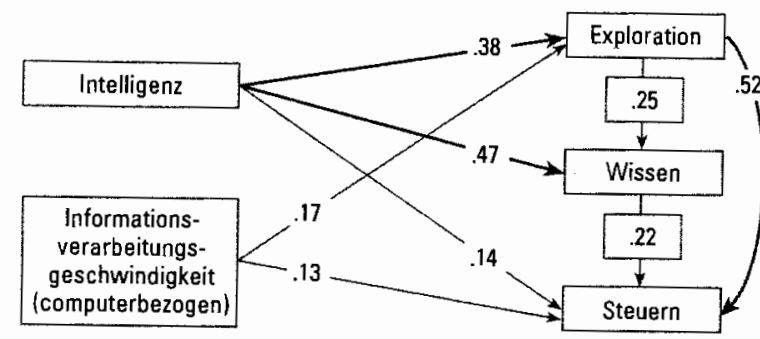

$\chi^{2}=2.51, d f=1, p=0.11$, RMSEA $=0.090$

Abbildung 2.1 Pfadmodell der Komponenten der Problemlösekompetenz beim Raumfahrtspie

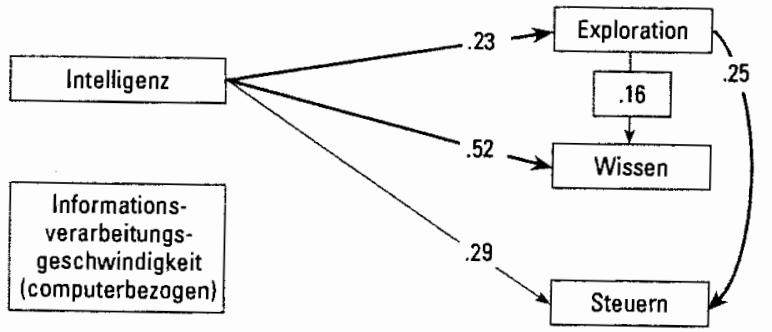

$x^{2}=7,17, d f=4, p=0.13$, RMSEA $=0.071$

Abbildung 2.2 Pfadmodell der Komponenten der Problenlösekompetenz beim Ökologie-Planspie 
deklaratives Wissen über Eigenschaften des simulierten Systems. Die beiden Pfadmodelle zeigen darüber hinaus neben einem direkten Pfad auch einen indirekten Pfad von Intelligenz über Exploration auf Steuern: Wer intelligent ist, exploriert effizient und erwirbt auf diese Weise prozedurales Wissen, das ihn über das deklarative Wissen hinaus befähigt, das System zielgerichtet zu steuern (vgl. Kröner, 200I). Bemerkenswert ist, dass das im Rahmen der Exploration erworbene deklarative Wissen beim Raumfahrtspiel dem erfolgreichen Steuern des Systems dienlich ist, was beim Ökologie-Planspiel jedoch nicht der Fall ist. Dies repliziert im Übrigen Befunde aus Untersuchungen, in denen das Ökologie-Planspiel als Lernumgebung im Geographieunterricht eingesetzt wurde (vgl. Leutner, I993; Leutner \& Schrettenbrunner, I989).

In den Pfadmodellen ist schließlich als Kontrollvariable die über eine Maus-TrackingAufgabe erfasste "Geschwindigkeit der Informationsverarbeitung" aufgenommen, die als basale Komponente der Intelligenz angesehen werden kann. Es zeigt sich, dass diese Variable zwar beim (mausgesteuerten) Raumfahrtspiel eine schwache Rolle spielt, nicht aber beim (tastaturgesteuerten) Ökologie-Planspiel. Sie kann also bei den weiteren Überlegungen vernachlässigt werden.

\section{Strukturmodell analytischer und dynamischer Problemlösekompetenzen}

\section{und Intelligenz}

Anhand der bisherigen korrelativen Analysen konnte gezeigt werden, dass die beiden Verfahren zur Erfassung dynamischer Aspekte der Problemlösekompetenz tatsächlich geeignet sind, den intelligenten Erwerb und die intelligente Anwendung von Wissen zu erfassen. Nun stellt sich die Frage, ob dynamisches und analytisches Problemlösen unterschiedliche Dimensionen der Problemlösekompetenz darstellen, die sich zudem von Intelligenz abgrenzen lassen.

Zur Klärung dieser Frage wurde auf der Basis von Rohkorrelationen eine konfirmatorische Faktoranalyse berechnet, bei der dynamisches Problemlösen, analytisches Problemlösen und Intelligenz als untereinander korrelierende latente Variablen spezifiziert wurden. Für das dynamische Problemlösen wurden die Explorations-, Wissens- und Steuerungskomponenten der beiden dynamischen Verfahren als Indikatorvariablen spezifiziert, für das analytische Problemlösen die drei verschiedenen Aufgabentypen, für Intelligenz der figurale und der verbale KFT-Subtest. Das Modell fittet die Daten gut und ist in Abbildung 2.3 dargestellt. Es zeigt sich, dass die drei Dimensionen zwar vergleichsweise hohe latente Korrelationen aufweisen $(.84<\mathrm{r}<.92)$, dass sie aber dennoch geeignet sind, die Korrelationsstruktur der Indikatorvariablen in angemessener Weise zu erklären. Dass die drei Dimensionen tatsächlich auch notwendig sind, belegten Modellrechnungen mit zwei Dimensionen bzw. einer Dimension, die in keinem Fall einen angemessen Fit ergaben.

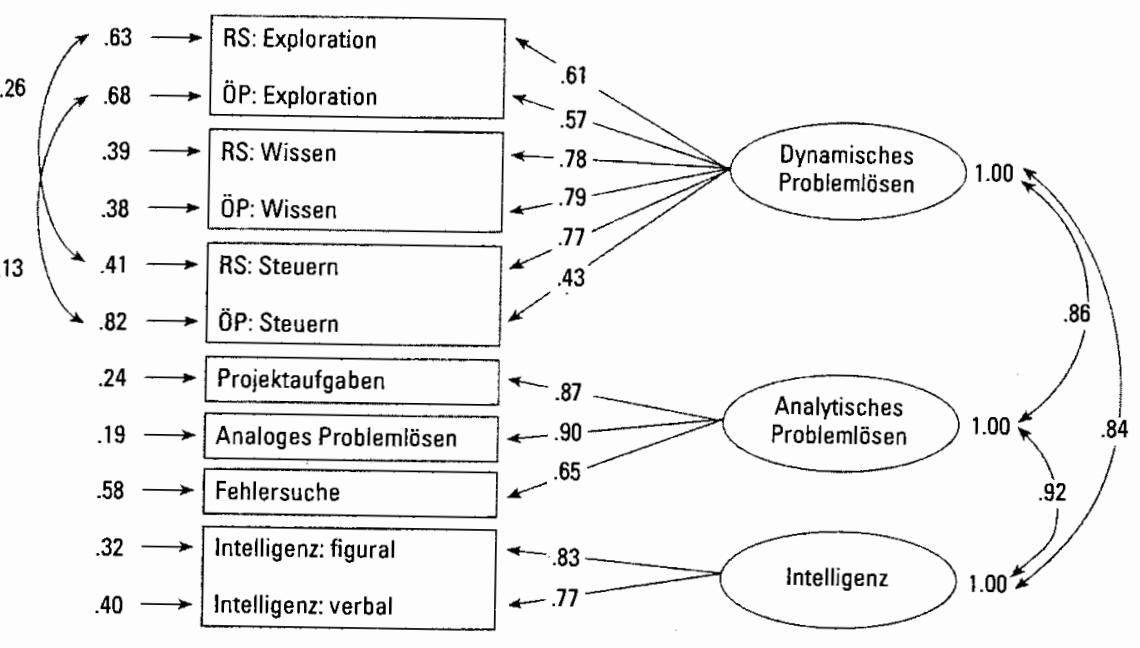

$\chi^{2}=46.79, \mathrm{df}=39, \mathrm{p}=0.18311, \mathrm{RMSEA}=0.045, \mathrm{RS}=$ Raumfahrtspiel, $\mathrm{OP}=$ Ökologie-Planspiel.

Abbildung 2.3 Strukturmodell der Problemlösekompetenzen und Intelligenz

\section{Strukturmodell der fachlichen und der Problemlösekompetenzen zusammen}

\section{mit Intelligenz}

In den Analysen zur Abbildung 2.3 konnte gezeigt werden, dass dynamisches und analytisches Problemlösen unterschiedliche Dimensionen der Problemlösekompetenz dar. stellen, die sich von Intelligenz abgrenzen lassen. Nun interessiert die Frage, ob sich die Dimensionen der Problemlösekompetenz auch dann noch voneinander abgrenzen lassen, wenn die fachlichen Kompetenzen bezüglich Lesen, Mathematik und Naturwissenschaften mit in das Modell aufgenommen werden. Abbildung 2.4 zeigt das passabel fittende Ergebnis (geringer dimensionale Modelle fitten deutlich schlechter). Es zeigt sich, dass sich die beiden Problemtypen auch in diesem umfassenden Kontext noch empirisch voneinander und von Intelligenz trennen lassen.

\section{Multidimensionale Skalierung der latenten Korrelation der PISA-Kompetenzen und der Intelligenz}

Tabelle 2.4 enthält die latenten Korrelationen der sechs Kompetenzdimensionen in Abbildung 2.4, und Abbildung 2.5 veranschaulicht die Struktur dieser Korrelationen in einer Art "Landkarte": Jede Kompetenz ist durch einen Punkt dargestellt: Je geringer der Abstand der Punkte, desto höher die latente Korrelation der betreffenden Kompetenzen. Es zeigt sich, dass die Kompetenzen eine seit Guttman (I954, 1957) so bezeichnete "Radex-Struktur" aufweisen: Intelligenz befindet sich im Zentrum der Struktur; die Kompetenzen sind gewissermaßen wie Tortenstücke als Sektoren um dieses Zentrum herum organisiert. Bemerkenswert ist, dass analytisches und dynamisches Problemlösen innerhalb ein und dessel- 


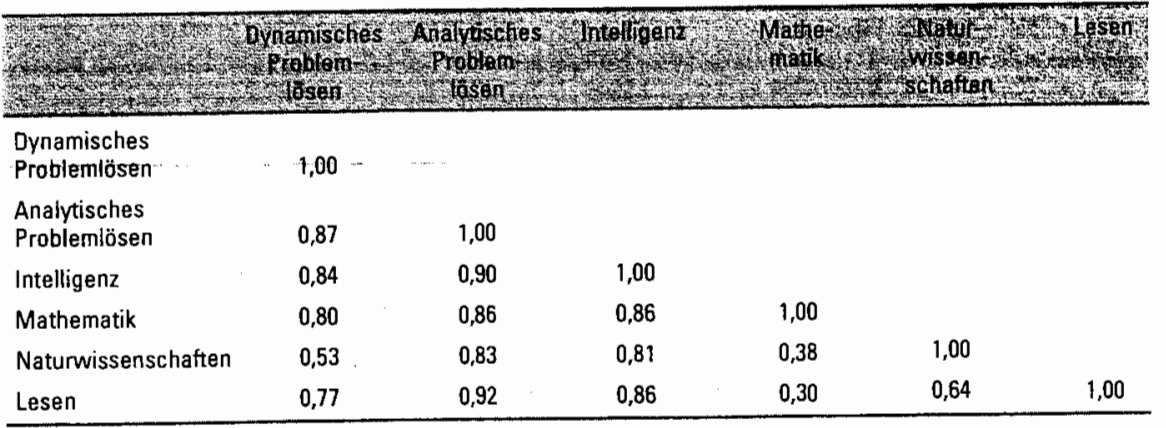

Tabelle 2.4 Latente Korrelation der Kompetenzdimensionen in Abbildung 2.4

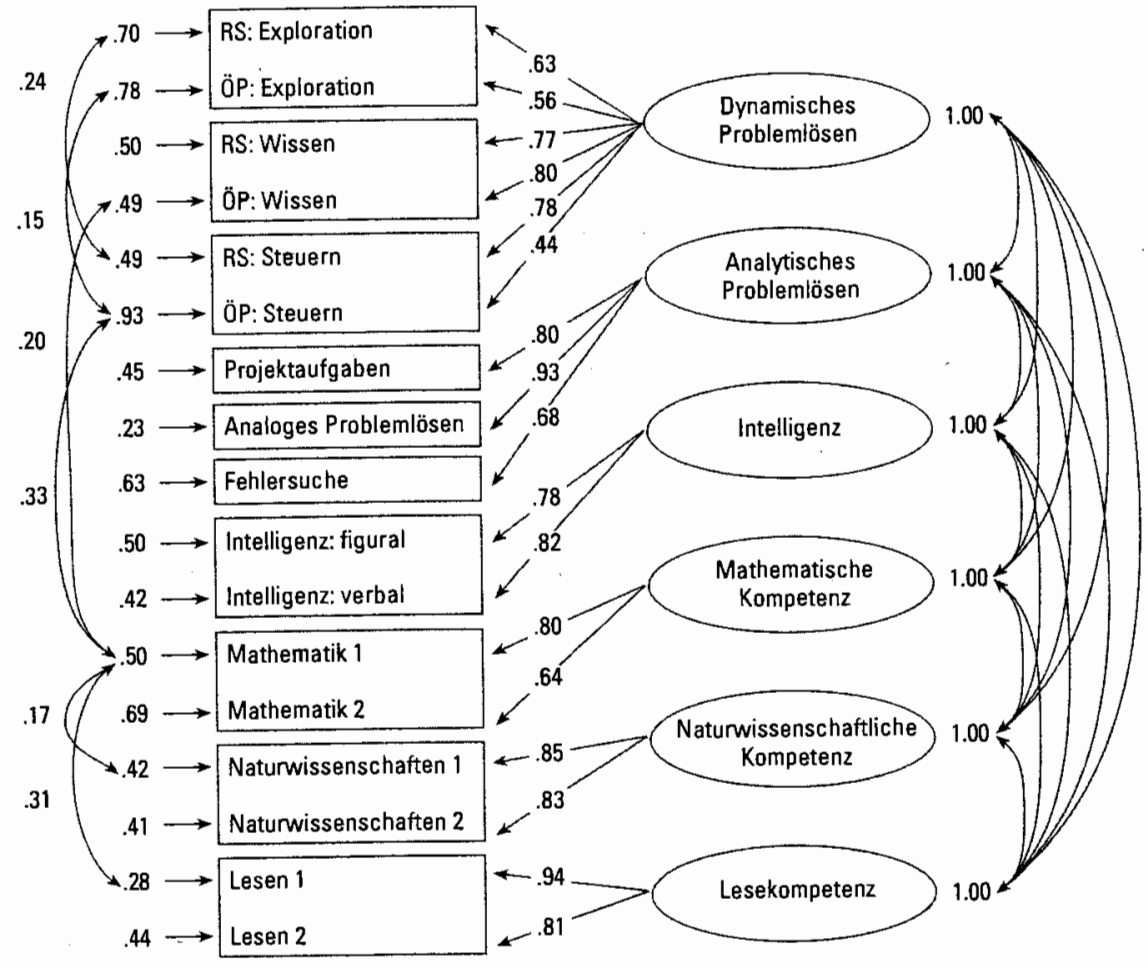

$\chi^{2}=129.84, \mathrm{df}=89, \mathrm{p}=0.01732, \mathrm{RMSEA}=0.057, \mathrm{RS}=$ Raumfahrtspiel, $\ddot{\mathrm{O}}=$ Ökologie - Planspiel.

Abbildung 2.4 Strukturmodell der im PISA-Test erfassten Kompetenzen und Intelligenz

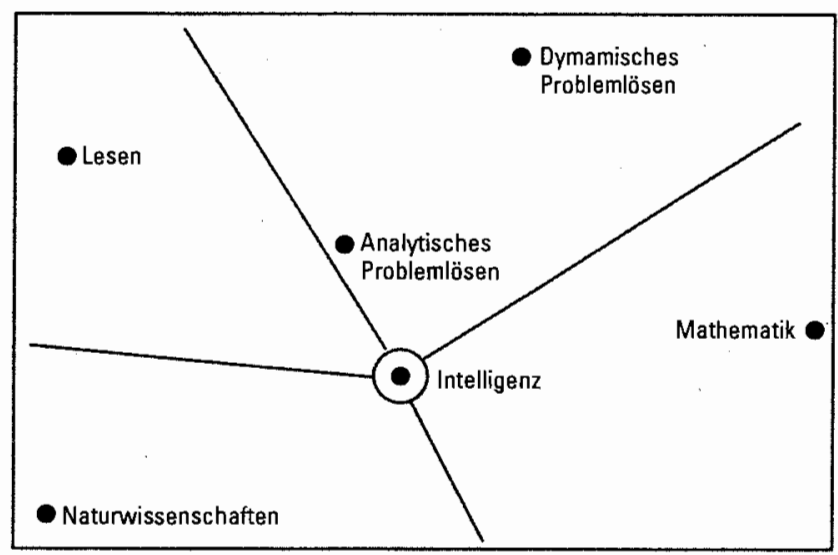

Abbildung 2.5 Nichtmetrische multidimensionale Skalierung der latenten Korrelationen aus Abbildung 2.4 und Tabelle 2.4 (fit stress $=.058$ )

ben Sektors liegen, der im Übrigen zwischen den Sektoren für Mathematik und Lesen platziert ist. Auffallig ist auch, dass analytisches Problemlösen weiter im Zentrum und damit näher an der Intelligenz liegt als alle anderen Kompetenzdimensionen. Dies verweist darauf, dass Intelligenz beim analytischen Problemlösen eine besondere Rolle spielt und dass mathematische Kompetenz und Lesekompetenz beim Problemlösen mehr ins Spiel kommen als naturwissenschaftliche Kompetenz. Für die konvergente und diskriminante Konstruktvalidität der Instrumente zur Erfassung fächerübergreifender Problemlösekompetenzen bedeuten diese Ergebnisse zusammenfassend Folgendes:

(I) Die computerbasierten Verfahren gehen über die schriftlichen Verfahren hinaus und erfassen nicht nur die intelligente Anwendung, sondern auch den intelligenten Erwerb von Wissen.

(2) Dynamisches und analytisches Problemlösen stellen unterschiedliche Dimensionen der Problemlösekompetenz dar, die sich von Intelligenz nicht nur theoretisch, sondern auch empirisch abgrenzen lassen.

(3) Die beiden Dimensionen der Problemlösekompetenz lassen sich auch dann noch voneinander und von anderen Kompetenzen abgrenzen, wenn die fachlichen Kompetenzen bezüglich Lesen, Mathematik und Naturwissenschaften mit betrachtet werden.

Die beiden Kompetenzen, analytische und dynamische Probleme zu lösen, erweisen sich damit - wie zu erwarten - als untereinander korrelierende Teilaspekte fächerübergreifender Problemlösekompetenz, die sich beide von fachlichen Kompetenzen in Lesen, Mathematik und Naturwissenschaften abgrenzen lassen. Darüber hinaus lassen sie sich ebenfalls von Intelligenz abgrenzen, wobei analytisches Problemlösen eine deutlich höhere Affinität zur Intelligenz zeigt als dynamisches Problemlösen. 


\subsection{Zusammenfassende Diskussion}

Ausgangspunkt der Erprobungsphase des PISA-2000-Problemlöse-Tests war die Frage nach der Praktikabilität einer Messung fächerübergreifender Problemlösekompetenzen im Large-Scale Assessment und den psychometrischen Eigenschaften der eigens entwickelten bzw. aus der Problemlösepsychologie adaptierten Instrumente. Die Ergebnisse zeigen, dass die psychometrischen Eigenschaften im Sinne von Schwierigkeit der Aufgaben und Reliabilität der Skalenwerte zufriedenstellend sind und dass die Instrumente, insbesondere auch die computerbasierten Instrumente, mit vertretbarem Aufwand eingesetzt werden können.

Ein weiteres Ziel der Erprobungsphase war es, die theoretisch relevante Frage nach der konvergenten und diskriminanten Konstruktvalidität der eingesetzten Instrumente zu beantworten. Die Ergebnisse zeigen, dass analytisches und dynamisches Problemlösen empirisch trennbare, aber konvergierende Aspekte einer fächerübergreifend konzipierten Problemlösekompetenz darstellen, die sich von theoretisch unterschiedlichen Kompetenzen wie allgemeiner Intelligenz im Sinne schlussfolgernden Denkens (Reasoning) und speziellen fachlichen Kompetenzen im Bereich des Lesens, der Mathematik und der Naturwissenschaften empirisch gut abgrenzen lassen.

Die Ergebnisse des Feldtests ermutigten das deutsche PISA-Konsortium, auch beim Haupttest des Jahres 2000 im Rahmen der nationalen Ergänzungen fächerübergreifendes Problemlösen zu erfassen, wobei sowohl analytische als auch dynamische Aspekte berücksichtigt werden sollten. Es wurden ein schriftliches Verfahren (die Projektaufgaben, siehe Kap. 3) und zwei computergestützte Verfahren (das Raumfahrtspiel und das Ökologie-Planspiel, Kap. 4) für den Einsatz in der Haupterhebung ausgewählt. Um den Gesamtbereich der Problemlösekompetenzen auch um einen sozialkommunikativen Aspekt zu erweitern, wurde zudem auf der Basis der Projektaufgaben eine Gruppenaufgabe konstruiert und gesondert vorerprobt, mit der im Haupttest 2000 die Fähigkeit zum kooperativen Problemlösen untersucht werden konnte (siehe Kap. 7).

\section{Anmerkungen}

I Mitglieder der Expertengruppe waren Joachim Funke, Detlev Leutner, Eckhard Klieme (Sprecher) und Peter Reimann, später auch Peter Frensch, sowie Joachim Wirth als Wissenschaftlicher Mitarbeiter.

2 Teststärkeanalysen hatten ergeben, dass 44 Beobachtungseinheiten pro Kombination ausreichen, um eine Korrelation der Größenordnung $r=.60$ mit einer Fehlerwahrscheinlichkeit erster und zweiter Art von jeweils .05 zu erkennen.
Eckhard Klieme, Johannes Hartig und Joachim Wirth

\section{Analytisches Problemlösen: Messansatz und Befunde zu} Planungs- und Entscheidungsaufgaben
Die nationale Erweiterung der PISA-Hauptstudie im Jahr 2000 unterschied bei dem Versuch, neben stärker fachbezogenen Kompetenzen auch fächerübergreifende Problem lösekompetenzen zu messen, zwischen analytischem und dynamischem Problemlösen. Beim analytischen Problemlösen sind die Ausgangslage und die sich daraus ergebende Problemstellung, oft unter Nutzung von Graphiken und Abbildungen, verbal beschrieben. Ziele sind wohldefiniert und dem Bearbeiter weitgehend klar. Alle notwendigen Informationen liegen dem Bearbeiter vor bzw. können aus vorhandenen Materialien (Texten, Tabellen, Graphiken) erschlossen werden. Um ausgehend von diesen Informationen Lösungen planen und Ziele erreichen zu können, benötigt man dennoch mitunter viele Zwischenschritte. Probleme - auch vom „analytischen“ Typus - lassen sich nich mit fertigen Routinen bewältigen. Man muss relevante Informationen auswählen oder erschließen, ein mentales Modell der Problemsituation erstellen, Alternativen durchspielen, Entscheidungen fällen und möglicherweise wieder korrigieren, Zwischenschritte und Ergebnisse laufend bewerten.

Die Bearbeitung eines Tests zum analytischen Problemlösen erfordert also durchaus komplexe Regulationsprozesse. Kriterium der Bewertung ist jedoch ausschließlich, ob die notwendigen Schlüsse letztlich erfolgreich vollzogen wurden, sodass die korrekte Lösung - wiederum verbal - angegeben bzw. unter vorgegebenen Alternativen identifiziert werden kann. Hierin liegt der wesentliche Unterschied zum dynamischen Problemlösen, wo der Bearbeiter in eine meist am Computer simulierte, zunächst intransparente Situation aktiv eingreift und dabei laufend beobachtet wird, sodass Kennwerte für die Güte des Explorationsprozesses, also des Wissenserwerbs, und der Wissensanwendung ermittelt werden können (vgl. hierzu Kap. 2 und 4).

Tests zum analytischen Problemlösen erfassen somit nicht unmittelbar die Güte von Problemlöseprozessen, sondern die Qualität der „Produkte“, das heißt der Lösungen, die durch schlussfolgerndes Denken und intelligente Nutzung von Wissen in weitgehend transparenten Situationen erzielt werden. Das Testergebnis soll die Fähigkeit messen, komplexe Informationen auszuwerten, zu verknüpfen und auf vorgegebene Ziele und Fragestellungen hin zu bündeln, um Handlungen zu planen und Entscheidungen zu fällen. 


\section{Problemlösekompetenz von Schülerinnen und schülern}

Diagnostische Ansätze, theoretische Grundlagen und empirische Befunde der deutschen PISA-2000-Studie 
Die Wiedergabe von Gebrauchsnamen, Handelsnamen, Warenbezeichnungen usw. in diesem Werk berechtigt auch ohne besondere Kennzeichnung nicht zu der Annahme, dass solche Namen im sinne der Warenzeichen- und Markenschutz-Gesetzgebung als frei zu betrachten wären und daher von jedermann benutzt werden dürften.

Umschlaggestaltung: KünkelLopka Medierientwicklung, Hejdelberg Druck und buchbinderische Verarbeitung: MercedesDruck, Berlin Gedruckt auf säurefreiem und chlorfrei gebleichtem Papier

Printed in Germany

ISBN 3-531-14736-6

\section{Einleitung}

Detlev Leutner, Joachim Funke, Eckhard Klieme und Joachim Wirth

1 Problemlösefähigkeit als fächerübergreifende Kompetenz

1.1 Einleitung: Fächerübergreifende Kompetenzen als Gegenstand von PISA

1.2 Der Kompetenzbegriff bei PISA

1.3 Problemlösen als Konzept und Forschungsgegenstand der kognitiven Psychologie

1.4 Problemlösen als Konzept und Forschungsgegenstand der differenziellen Psychologie

1.5 Analytische und dynamische Aspekte des Problemlösens

1.6 Selbstregulation beim dynamischen Problemlösen

Detlev Leutner, Joachim Wirth, Eckhard Klieme und Joachim Funke

2 Ansätze zur Operationalisierung und deren Erprobung in Feldtest zu PISA 2000

2.1 Einleitung

2.2 Operationalisierung von Problemlösekompetenz

2.3 Empirische Erprobung im Feldtest

2.4 Zusammenfassende Diskussion

Eckhard Klieme, Joharnes Hartig und Joachim Wirth

3 Analytisches Problemlösen: Messansatz und Befunde zu Planungsund Entscheidungsaufgaben

3.1 Projektaufgaben: Konzeption und Auswertungsstrategie

3.2 Validität und Niveaustufen des analytischen Problemlösens

3.3 Analytische Problemlösekompetenz in Abhängigkeit von Schulform, Bundesland und Geschlech

3.4 Fazit

Joachim Wirth und Joachim Funke

4 Dynamisches Problemiösen: Entwicklung und Evaluation eines neuen Messverfahrens zum Steuern komplexer Systeme

4.1 Einleitung

4.2 Instrument und Leistungsmaße 